\section{Cureus}

\title{
May-Thurner Syndrome Variant Identified in a Cadaver: First Reported Case
}

Emily A. Simonds ${ }^{1}$, Mayank Patel ${ }^{2}$, Marc Vetter ${ }^{1}$, Joe Iwanaga ${ }^{3}$, Rod J. Oskouian ${ }^{4}$, R. Shane Tubbs ${ }^{5}$

1. Seattle Science Foundation, Seattle, USA 2. Clinical Anatomy Research, Seattle Science Foundation, Seattle, USA 3. Medical Education and Simulation, Seattle Science Foundation, Seattle, USA 4. Neurosurgery, Swedish Neuroscience Institute, Seattle, USA 5. Neurosurgery, Seattle Science Foundation, Seattle, USA

$\square$ Corresponding author: Emily A. Simonds, easimonds@gmail.com Disclosures can be found in Additional Information at the end of the article

\section{Abstract}

May-Thurner syndrome (MTS) is defined as the compression of the left common iliac vein by the right common iliac artery. Herein, we describe an unusual case of a male cadaver with rightsided compression of the inferior vena cava and the left and right common iliac veins by the right common iliac artery. This is an unusual variant of this syndrome and the first known case report. We suggest this variant be termed MTS type II due to the additional compression of the inferior vena cava.

Categories: Cardiac/Thoracic/Vascular Surgery, General Surgery, Other

Keywords: may-thurner syndrome, cockett syndrome, iliac vein compression syndrome, iliocaval compression syndrome, common iliac artery, plaques, artherosclerotic

\section{Introduction}

May-Thurner syndrome (MTS) is a rare disorder defined as obstruction caused by chronic left common iliac vein compression by the overlying right common iliac artery [1]. MTS is also known as iliac vein compression syndrome and is one of several pathologies included in the disease profiles known as nonthrombic iliac vein lesions. Such compression of the left common iliac vein by the overlying right common iliac artery inhibits venous blood return from the lower extremity and pelvis [1]. Variants of MTS include isolated compression of the right iliac vein or inferior vena cava (IVC) [2-3]. We present an unusual variant of MTS where the inferior vena cava and left and right common iliac veins were all compressed by an atherosclerotic right common iliac artery in a cadaver.

Received 03/22/2018 Review began 03/26/2018 Review ended 03/28/2018 Published 03/30/2018

\section{(C) Copyright 2018}

Simonds et al. This is an open access article distributed under the terms of the Creative Commons Attribution License CC-BY 3.0., which permits unrestricted use, distribution, and reproduction in any medium, provided the original author and source are credited.

\section{Case Presentation}

During the routine dissection of the abdominopelvic region of a fresh frozen African American male cadaver (74 years old at death), the right common iliac artery was observed to be atherosclerotic and deviated more superiorly than normal (Figure 1). This resulted in simultaneous compression of the distal inferior vena cava and the left and right common iliac veins. The aorta bifurcated at the L4 vertebra and the inferior vena cava bifurcated at the L5 vertebral body. In the supine position, venous stasis was obvious in the prestenotic part of the inferior vena cava (Figure 1). Digital pressure of this engorged segment was not sufficient to propel venous blood within the inferior vena cava distally. Blood flow was only possible with anterior displacement of the overlying and atherosclerotic right common iliac artery, thus illustrating the compressive nature of the overlying right common iliac artery. No obvious signs 


\section{Cureus}

of lower limb or pelvic visceral edema were noted. The diagnosis of an MTS variant was made, i.e., not only was the left common iliac vein compressed, but also the distal inferior vena cava and right common iliac vein.

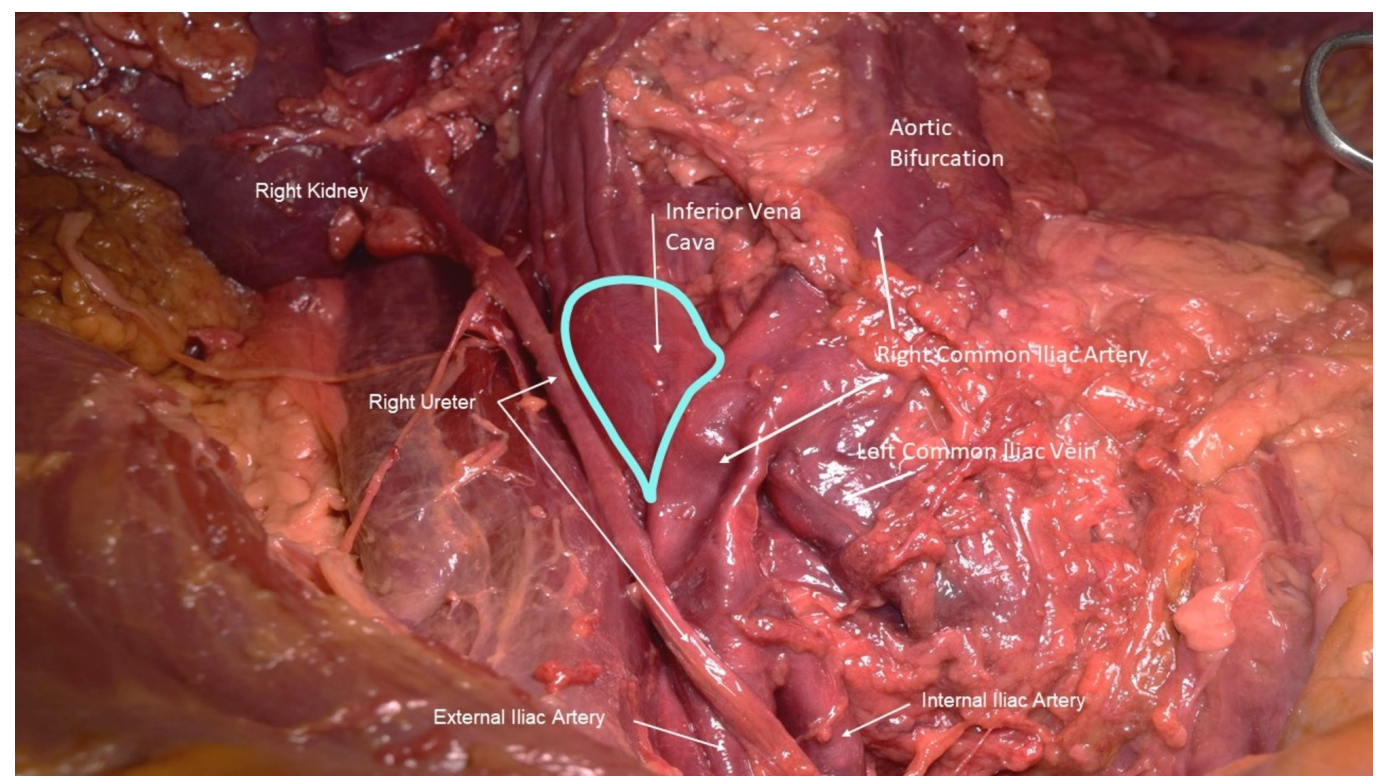

\section{FIGURE 1: Anterior dissection of male cadaver with MTS variant}

The right common iliac artery is shown crossing over the anterior surface and compressing the distal inferior vena cava and left common iliac vein. The right common iliac vein (not shown) is also compressed. Note the dilated distal part (outlined in teal) of the inferior vena cava. Also, note the dimpling of the right common iliac artery due to atherosclerotic plaque within this vessel. The left common iliac artery is not shown.

\section{Discussion}

To our knowledge, this is the first known case report of a MTS variant in a cadaver. Patients with MTS may present with discomfort in their left lower extremities due to pain, swelling, and deep vein thrombosis (DVT) [1]. One of the limitations of this case report is that the presence of these clinical symptoms are unknown in our specimen. However, the findings from our dissection are indicative of MTS. Pain from venous claudication after exercise occurs in $85 \%$ of symptomatic MTS patients [4].

The prevalence of this disease is also unknown, but it is estimated that $2-5 \%$ of all lower extremity venous disease can be attributed to MTS, which occurs more often in women than men [1]. MTS often goes undiagnosed and may only become symptomatic when there is trauma, or a significant surgery such as knee or hip replacement is involved. There are several rare presentations which can occur such as acquired MTS after endovascular stent/graft procedures [5-6]. MTS may present secondary to urinary bladder distention [7]. MTS may also play a role in spontaneous left iliac vein rupture due to spontaneous retroperitoneal hematoma in women with lower extremity DVT [8].

Clinical management of MTS includes compression stockings, evaluations by vascular surgeons, cardiology, and interventional radiology. Endovascular stenting of the iliac veins or open repair may be necessary [9]. 


\section{Conclusions}

The case reported herein is unusual. To our knowledge, this is the first case of such a variant identified in a cadaveric specimen. We suggest this variant be termed MTS type II due to the additional compression of the inferior vena cava. Clinicians must consider MTS as a possible diagnosis when patients present with acute pain or swelling in the lower extremities.

\section{Additional Information}

\section{Disclosures}

Human subjects: Consent was obtained by all participants in this study. Conflicts of interest: In compliance with the ICMJE uniform disclosure form, all authors declare the following:

Payment/services info: All authors have declared that no financial support was received from any organization for the submitted work. Financial relationships: All authors have declared that they have no financial relationships at present or within the previous three years with any organizations that might have an interest in the submitted work. Other relationships: All authors have declared that there are no other relationships or activities that could appear to have influenced the submitted work.

\section{References}

1. Brazeau NF, Harvey HB, Pinto EG, Deipolyi A, Hesketh RL, Oklu R: May-Thurner syndrome: diagnosis and management. Vasa. 2013, 42:96-105. 10.1024/0301-1526/a000252

2. Fernando RR, Koranne KP, Schneider D, Fuentes F: May-Thurner syndrome in a 68-year-old woman after remote abdominal surgery. Tex Heart Inst J. 2013, 40:82-87.

3. Fretz V, Binkert CA: Compression of the inferior vena cava by the right iliac artery: a rare variant of May-Thurner syndrome. Cardiovasc Intervent Radiol. 2010, 33:1060-1063. 10.1007/s00270-009-9671-y

4. Delis KT, Bountouroglou D, Mansfield AO: Venous claudication in iliofemoral thrombosis: long-term effects on venous hemodynamics, clinical status, and quality of life. Ann Surg. 2004, 239:118-126. 10.1097/01.sla.0000103067.10695.74

5. Hermany PL, Badheka AO, Mena-Hurtado CI, Attaran RR: A unique case of May-Thurner syndrome: extrinsic compression of the common iliac vein after iliac artery stenting. JACC Cardiovasc Interv. 2016, 9:39-41. 10.1016/j.jcin.2015.11.042

6. Pandit AS, Hayes M, Guiney-Borgelt S, Dietzek AM: Iatrogenic May-Thurner syndrome after EVAR. Ann Vasc Surg. 2014, 28:739-717. 10.1016/j.avsg.2013.07.019

7. Hung JB, Hsu CW, Tsai SH: Prostatism and May-Thurner syndrome. Am J Emerg Med. 2013, 31:445-441. 10.1016/j.ajem.2012.05.029

8. Hosn MA, Katragunta N, Kresowik T, Sharp WJ: May-Thurner syndrome presenting as spontaneous left iliac vein rupture. J Vasc Surg Venous Lymphat Disord. 2016, 4:479-481. 10.1016/j.jvsv.2016.03.007

9. Greer DM, Buonanno FS: Cerebral infarction in conjunction with patent foramen ovale and May-Thurner syndrome. J Neuroimaging. 2001, 11:432-434. 10.1111/j.1552-

6569.2001.tb00074.x 